\title{
Machine Learning, Deep Learning and Artificial Intelligence approach for predicting CRISPR for the Cancer treatment
}

\author{
Dr. Anuraj Nayarisseri \\ Email:anuraj@eminentbio.com \\ Tel:+91 9752295342
}

1. In silico Research Laboratory, Eminent Biosciences, 91, Sector-A, Mahalakshmi Nagar, Indore - 452010, Madhya Pradesh, India.

2. Bioinformatics Research Laboratory, LeGene Biosciences Pvt. Ltd. Indore - 452010, Madhya Pradesh, India.

\section{Background}

Cancer forms second-most cause of death in the USA next to cardio vascular diseases, contributing to significant economic and social burden. WHO reports suggest that, almost 18 millions cancer cases and 60 lakhs deaths from cancer is expected in the US in 2019 alone. The most common types such as lung, breast, prostate, and colorectal cancer are mostly prevalent in the United States and worldwide. Although knowledge of molecular diagnosis has improved in the recent years, advanced therapeutic approaches still needs to be addressed for the diseases like cancer. In the recent years, CRISPR Cas9 system has emerged as a powerful tool for genome editing in mammalian cells including humans which offers a great promise in cancer therapeutics. For instance, the immune cell can be modified to express certain antigenic receptors like CARs (Chimeric Antigen Receptors), which efficiently recognizes and kill cancer cell. CARs have shown to be faster and effective in killing tumour cells than any immune cells studied so far. The immune cell(T-cell) can be isolated from the venous blood and be genetically modified to effectively target cancer cells. CRISPR technology can be deployed to edit and insert T-Cell CAR at the target location of the genome where the variants occur or tumour gene identified. The study hence proposes the creation of highly potent $T$ endowed with CAR expression, which can be further inserted in the patient's genome with retroviral delivery methods. 
Next Generation Sequencing technology with targeted amplicon methods can be used to detect on-and off-target mutations and further establish phenotype-genotype relations. Several trained libraries and machine learning algorithms, such as Delphi, are available to predict a CRISPR cleavage and editing patterns. During the CRISPR genome editing, the endonucleases such as cas9 assisted by guide RNA, cleaves the target DNA. In the process, genetic material is stitched back together, later it may be inaccurate and in the absence of the template this process may loose its precise targeting. Artificial intelligence can therefore be deployed for faster and accurate CRISPR enabled genome editing, overcoming the pitfalls of non-specific genome targeting.

The main goal of precision cancer medicine is the accurate prediction of finest drug therapies depends on the personalized genetic profiles of patient tumors. Ideally, such predictions are depends on well-established genetic cause-and-reflex association that are disrupted in cancer cells. According to a statistical survey done at Harward medical school, In USA almost 17 various research studies were done using CRISPR to treat cancer. But most of those studies were used by genome editing technology to engineer immune cells to attack tumor cells. But recently researchers in china were rumored that they were directly use CRISPR to knock out viruses that cause cervical cancer which is not been done in yet.

The present research proposes a machine learning approach to exploit the large scale genomic data by NGS. The machine learning and deep learning methods can be effectively used to identify and determine the tumor immunogenicity which may pave for the development of a scoring system (immunophenoscore IPS algorithm) for immunogenicity quantification, hence, facilitatating for precision immuno-oncology therapy. The machine learning method can be also be used to classify the immune cell subtype based on gene set enrichment analysis. With random forest classification in machine learning and deep learning approaches, the study can further determine the immune checkpoint blockade response phenotypes of melanoma patients. The present study, hence, is pursued to apply machine learning algorithms for predicting CRISPRCas9 cleavage patterns concerted with CARs mechanism, which can be anticipated to provide better long-term prognosis of cancer patients in comparison to tumor grade. 